\title{
URBANISMO, CIENCIA Y EVOLUCIÓN
}

\author{
Felipe Colavidas Espinosa (Dr. Arquitecto - Profesor titular DUyOT)
}

Para Paco Alonso; desde luego, por lo que dice, pero, sobre todo, por lo que hace: por la materialización de lo dicho.

La piedra angular del método científico es el postulado de la objetividad de la Naturaleza. Es decir, la negativa sistemática a considerar capaz de conducir a un conocimiento "verdadero" toda interpretación de los fenómenos dada en términos de causas finales, es decir de "proyecto". Se puede datar exactamente el descubrimiento de este principio. La formulación, por Galileo y Descartes, del principio de inercia, no fundaba sólo la mecánica, sino la epistemología de la ciencia moderna, aboliendo la física y la cosmología de Aristóteles.

Jacques Monod

Tener ideología no es tener ideas. Estas no son como las cerezas, sino que vienen sueltas, hasta el punto de que una misma persona puede juntar varias que se hallan en conflicto unas con otras. Las ideologías son, en cambio, como paquetes de ideas preestablecidos, conjuntos de tics fisionómicamente coherentes, como rasgos clasificatorios que se copertenecen en una taxonomía o tipología personal socialmente congelada.

Rafael Sánchez Ferlosio

En la atenta invitación de José Fariña a participar en el número conmemorativo de $\mathrm{CI}[\mathrm{ur}$ ], se manifiesta la intención de que esta centésima entrega de la revista sea, "solo por una vez", un número de opinión. Se expresa así la convicción de que el hecho de opinar es excepcional en la larga trayectoria de este foro de investigación urbanística.

Sin entrar ahora a validar o rebatir esta opinión, es irrefutable que, desde una dimensión estrictamente científica, la contraposición entre hechos y opinión resulta determinante. Ciertamente, el enfrentamiento ciencia versus creencia dividió radicalmente en dos el conocimiento humano, que había tenido su origen genuino en Grecia. El esencial descubrimiento de esta confrontación se debe a la ciencia nueva barroca -con Galileo, Bacon, Descartes y Newton a la cabeza- y al posterior acento empirista de la Ilustración escocesa. Como no podía ser de otra manera, esta nueva epistemología determina también el devenir del urbanismo.

De esto es de lo que preferentemente quiero tratar aquí ${ }^{1}$, pues en clara

${ }^{1}$ El contenido esencial de este artículo surge de mi antiguo interés intelectual -el que, en cierta forma, impulsó la redacción de mi tesis doctoral La ciudad pensada, 1990- por la comprensión completa de la habitabilidad general como hecho central de nuestro linaje. Desde hace años, vengo realizando con Isabel Bravo (arquitecta, ETSAM, 1995) un Tratado de habitabilidad, en redacción, algunas de cuyas cuestiones cruciales (la habitabilidad como elemento exógeno clave de la constitución y progresivo desarrollo de la naturaleza humana; su reconocimiento como hecho sensible material determinante de 
confrontación con el auténtico conocimiento científico, tengo a la sobreabundancia de ideología y opinión por el rasgo negativo más sobresaliente del urbanismo: el que más lastra y debilita su capacidad de clarificar la habitabilidad humana y de enfrentarse con la máxima eficacia a la mejora del territorio construido ${ }^{2}$. Este asunto no solo afecta a los tres últimos decenios, que esta revista nos acota como ámbito temporal de análisis, sino al tiempo transcurrido desde que se intentó por primera vez fundamentar la autonomía disciplinar, con las primeras propuestas rigurosas de creación de nueva ciudad (Cerdá, 1859) y de reestructuración de la existente (Haussmann, 1856). Y es que fue entonces cuando se trató de esbozar de forma sistemática el paso de una habitabilidad espontánea a otra más ordenada que, al hilo de esta nueva epistemología científica, aspiraba a materializarse bajo el principio de objetividad y la metodología propiciada por la experiencia y la lógica.

La habitabilidad espontánea ha dominado tanto nuestro pasado prehistórico nómada como el proceso histórico de urbanización que, con origen en la ciudad de las primeras civilizaciones, se ha desplegado en un elenco fáctico de morfologías urbanas universales -construidas sustantivamente sin proyecto- ${ }^{3}$ hasta desembocar en este intento novecentista de planificar la urbanización. Pero este primer esbozo científico/disciplinar, en lugar de haberse consolidado, está aún pendiente de realización. También la habitabilidad está lejos de haberse afirmado como categoría científica autónoma: en un sentido global, es el objeto de estudio específico del urbanismo y de la ordenación del territorio ${ }^{4}$, en la doble vertiente de primera gran función y de estructuras físicas que constituyen su soporte material construido. Se define como el conjunto de cualidades que adquiere todo territorio natural al ser transformado en un lugar verdaderamente ordenado y lleno de significación, útil al desarrollo de las diversas funciones y actividades que, en torno a las dos esenciales de producción y residencia, precisa la vida humana para garantizar su reproducción saludable y el morar próspero.

Por pertenecer también al campo de las artes, el urbanismo se extiende más allá de la dimensión empírica y positiva; y, por tanto, los procedimientos lógicos y experimentales han de ser, en muchos casos, compartidos con el método analógico en la vertiente del urbanismo-arte. Pero la dimensión objetiva del urbanismo es de

su existencia, o la reivindicación de su rango y categoría científica) quedan apuntadas en el presente texto. Parte singular, sustantiva e indisoluble de esa habitabilidad general es la habitabilidad básica, que inicié como materia independiente de docencia en el año 92 en el DUyOT-ETSAM, a la que se incorporó unos años después Julián Salas, y en la que ahora ultiman sus trabajos específicos tres doctorandos (Carlos Galán, Belén Gesto y Luis Perea) y también sus diversos proyectos y actividades algunos otros miembros destacados de nuestro grupo de CD-HaB-ETSA-UPM.

${ }^{2}$ Tengo a este urbanismo-ideología, de carácter especulativo, por el dominante, pero no único, claro; obviamente hay también otras formas honrosas de hacer urbanismo, que priman fundamentar sus conclusiones en datos y que nunca han faltado en la disciplina, aunque a mi juicio hayan sido siempre corrientes minoritarias.

3 Sin menoscabo de que, a efectos de su universalidad, sea preciso matizar el sesgo occidental que manifiestan las etapas finales (precisamente, las que surgen a partir del Renacimiento y la ciencia barroca) de esta sucesión de formas urbanas históricas que se sucedieron hasta la inauguración de la disciplina en el XIX, se identifica el siguiente elenco: ciudad arcaica, clásica, medieval, gótica, renacentista, barroca, neoclásica e industrial. Estos modelos históricos hacen referencia tanto a ciudades completas como a tejidos parciales de ellas.

${ }^{4} \mathrm{Y}$ del conjunto de otras muchas disciplinas que, como la ingeniería o la arquitectura, conciernen a la totalidad del sector de la construcción mundial. 
tal envergadura que resulta prioritario su sometimiento al arsenal metodológico de todas las ciencias, caracterizado por ser fáctico, racional, sistemático, objetivo, analítico y empírico ${ }^{5}$. En este sentido, llama la atención que no exista siquiera un acuerdo universal sobre la analítica rigurosa del espacio urbano ni sobre el consecuente glosario básico de términos que habrían de constituir la base instrumental necesaria mediante la que poder abordar los dos diferentes cometidos que está obligado a desempeñar la disciplina: el conocimiento teórico, ajustado al principio de objetividad, y el profesional, de intervención práctica sujeta a dicho conocimiento.

En el ámbito de la compresión, la tarea pendiente es proporcionar una imagen mental completa de la habitabilidad y de su crucial importancia en el desarrollo de nuestro linaje. Este conocimiento teórico precisa también ser abordado en su vertiente pragmática. Al hilo de promover el trabajo empírico, planteo aquí instituir una categoría específica -dentro de la más amplia de condiciones generales de vida, vigente en las ciencias sociales y humanas- que, bajo la denominación índice de habitabilidad, sintetice numéricamente tales condiciones en cada país o territorio, de manera similar a como lo hacen, en sus respectivas materias, el índice de desarrollo humano y la renta per cápita.

Me parece oportuno recordar aquí una de las más trascendentales polémicas ocurridas en el mundo de la cultura y la ciencia: la suscitada por la conferencia que C.P. Snow pronunció en Cambridge en 1959. En ella, se denunciaba la división del pensamiento occidental en dos culturas: una literaria o humanística y otra científica, separadas ambas por una barrera de desconocimiento y prejuicios mutuos; barrera a la que Snow achacaba muchas de las dificultades que ralentizan el progreso fáctico. Se abría así camino a la tercera cultura, acuñada por J. Brockman en $1995^{6}$. Es a este territorio donde urge llevar al urbanismo desde la primera cultura en la que mayoritariamente aún sigue ubicado: una posición no empírica, plagada de ideología, que a menudo confunde el análisis de los hechos con la adhesión voluntarista a principios mítico-abstractos. Para tratar de instituir la habitabilidad como categoría científica, el reto está en lograr incardinar el urbanismo dentro de la teoría darwiniana de la evolución -más en concreto, dentro del neodarwinismo ${ }^{7}-$ : el modelo explicativo unificado más veraz que, quizá, ha existido nunca.

Es en dicha teoría explicativa donde la habitabilidad se constituye, en cuanto medio y entorno artificial, como un concepto exclusivo de nuestro linaje humano. Si el resto de las especies se adaptan sustantivamente sin proyecto al medio natural, la nuestra es la única que adapta, sustantiva y voluntariamente, el medio a sus propios intereses. Es en esta condición de entorno artificial donde la habitabilidad destaca

\footnotetext{
5 Tomo así distancia de otro tipo de propuestas especulativas que nunca han sido -al menos, en lo que yo conozco- mínimamente desarrolladas más allá de su nominación, como la de un posible método holístico.

6 También a los trabajos de A. Sokal y J. Bricmont sobre Imposturas intelectuales y a los de la institución Edge: un territorio en el que se reconoce a los científicos y los pensadores del mundo empírico su papel para explicar significados vitales profundos que antes se habían dejado exclusivamente en manos de los intelectuales tradicionales, de sesgo humanístico.

7 También denominada Síntesis Moderna de la Evolución, nacida en los años treinta del pasado siglo y afianzada con los avances actuales en genética.
} 
como el hecho más notorio de la producción tecnológico-cultural ${ }^{8}$ de nuestra especie.

Al efecto de poder llegar a entender este ajuste del medio natural al sujeto, habremos de situarnos en el clado que, hace unos siete millones de años, y al adoptar la postura erguida, nos distinguió del linaje filogenéticamente hermano. El bipedalismo, al dejar libres las manos, hizo posible el cambio alimenticio y propició la puesta en marcha del crecimiento estructural del cerebro, con todas sus consecuencias. Es en este marco conceptual donde la habitabilidad encuentra su base objetiva para poder alcanzar el mencionado rango científico. $Y$ es que, en cuanto entorno artificial, la habitabilidad constituye uno de los factores clave del éxito del Homo sapiens, pues resulta el elemento determinante tanto de la baja ininterrumpida de las tasas de mortalidad como, consecuentemente, del crecimiento demográfico.

Por otra parte, la habitabilidad constituye, en cuanto construcción del territorio, la columna vertebral económica del desarrollo material, y por ello, de la producción y el consumo de riqueza. Concierne a la reproducción de la propia fuerza de trabajo -a través de la residencia- y a la de las mercancías -en la producción propiamente dicha-, que, en cuanto es inversión en trabajo y capital fijo, viene transformando de forma sustantiva la superficie del planeta. Sin embargo, hay otra vertiente de la habitabilidad que trasciende lo material: es la capacidad de ese espacio construido para re-obrar sobre lo humano y hacernos, literalmente, a nosotros mismos. En cuanto constitutiva de civilización (ciudad) y cultura, la habitabilidad es capaz de transformar moral y físicamente a las personas. Ambos desarrollos quedan magistralmente recogidos en esta temprana intuición de Aristóteles: "La ciudad tiene su origen en la urgencia del vivir, pero persiste para el vivir bien, para la vida buena".

No quiero cerrar estas líneas sin reseñar un aspecto positivo del urbanismo en estas últimas décadas: con la revolución que ha supuesto el desarrollo de herramientas como Google Earth o Street View es posible disponer ya de información en tiempo real de planimetría de lugares remotos. En el ámbito de la enseñanza, especialmente en las asignaturas relacionadas con el desarrollo de habitabilidad básica, se ha hecho posible así el trabajo sobre territorios de los que hace tan solo unos lustros habría resultado muy costoso conseguir información. Esto supone una aportación crucial para realizar la elección adecuada del sitio de asentamiento: el primer y más importante de los cuatro pasos canónicos del proceso de urbanización expresado en la teoría de la habitabilidad ${ }^{9}$ que formalicé hace unos años, ampliando una vieja propuesta de Manuel Solá-Morales.

Más allá de su carácter operativo, cabe decir que este conjunto de herramientas nacidas de la revolución tecnológica ha transformado la médula de la disciplina, facilitando la consecución de una metodología propia de la ciencia: la primera aspiración de la que este escrito ha querido dejar constancia.

\footnotetext{
8 Agustín García Calvo definió el lenguaje como la Primera Gran Máquina que, en el ámbito mental, inmaterial, permite a la especie Homo sapiens construir el resto de máquinas. Al hilo, resulta fructífero considerar que la ciudad es la Segunda Gran Máquina, la más potente y determinante desde el punto de vista material, fáctico.

9 Las cuatro etapas canónicas de la teoría de la habitabilidad: 1) Elección del suelo adecuado, 2) Parcelación del primer orden que establece la división urbana en público y privado; 3) Urbanización del espacio público (RELP) y 4) Edificación de las parcelas residenciales y de dotaciones y equipamientos.
} 